\title{
Characterization of Intra-abdominal Tissues from in vivo Animal Experiments for Surgical Simulation
}

\author{
Jung Kim ${ }^{1}$, Boon K. Tay ${ }^{1}$, N. Stylopoulos ${ }^{2}$, D.W. Rattner ${ }^{3}$, and M.A. Srinivasan ${ }^{1}$ \\ The Touch Lab, \\ ${ }^{1}$ Department of Mechanical Engineering, Massachusetts Institute of Technology, MA, USA \\ jungkimamit.edu \\ ${ }^{2}$ Department of Surgery, Massachusetts General Hospital, Boston, MA 02114 \\ ${ }^{3}$ Division of General and Gastrointestinal Surgery, \\ Massachusetts General Hospital, Boston, MA 02114
}

\begin{abstract}
The lack of data on in vivo material properties of soft tissues is one of the impediments in the development of realistic surgical simulators. The measurement of these properties is not a trivial task, due to the difficulty of the testing itself and the complexity of tissue mechanical behavior. We have developed a system for measuring the mechanical properties of soft tissues in vivo using a robotic device fitted with a force transducer. We measured the response of soft tissues in intra-abdominal organs including the liver and lower esophagus of pigs by using both static and dynamic indentations. We characterize these properties using nonlinear models as well as a linear model. These material models can be effectively integrated into a simulator to provide the user with realistic visual and haptic feedback.
\end{abstract}

\section{Introduction}

A Virtual Reality (VR)-based surgical simulation system [1, 2], which provides an innovative tool for training of medical personnel, requires accurate modeling of soft tissue properties as well as fast computation algorithms to simulate the tissue deformation induced by a surgical tool. While the computation algorithms have been widely studied by many research groups, the characterization of the tissue properties, especially in vivo properties, has not been sufficiently investigated due to difficulty of testing and complex tissue behavior such as nonlinearity and rate-dependency.

Several studies have been carried out in characterizing the mechanical behavior of the various biological tissues in vitro [3] [4]. However, these data may not be suitable for surgical simulation because the mechanical properties of soft tissues change after death. After removing samples from the living state, the conditions of tissues change drastically from factors such as 1) temperature (changes in viscosity) 2) hydration (drying up might change elasticity and viscosity) 3) break-down of proteins (change in stiffness) 4) loss of blood supply. Moreover, the boundary conditions of a sample are different from in vivo states and therefore its force-displacement relationship will change when the sample is cut away from the rest of the organ. Recently, considerable research has been done on measurements of mechanical properties of tissues in vivo [5-8]. For example, Ottensmeyer [9] designed a device named TeM 
PeST 1-D to measure organ mechanical properties. From experiments on pig livers, he measured the in vivo stiffness over various frequencies with a maximum amplitude $500 \mu \mathrm{m}$ and a $100 \mathrm{~Hz}$ bandwidth. Another example is the Dundee Single Point Compliance Probe [6], which is designed to measure static force responses of organs against indentation stimuli during an open surgery. As a hand-held probe, it can indent up to a maximum depth of $6 \mathrm{~mm}$ and can measure the corresponding reaction forces. Force-displacement curves of the organs can be produced from the corresponding data and stiffness of the tissues can be inferred. It has been used to obtain force-displacement relationship data on the human liver.

Although useful, the measurements in previous work cited above are not entirely satisfactory for our application in the sense that the indentation depth, frequency bandwidth and boundary conditions that those devices are able to deliver are different from those relevant to typical laparoscopic procedures. Tendick et al. measured in vivo material properties of a pig small intestine by using various kinds of devices they developed [7]. Our group has been performing animal experiments to characterize in vivo soft tissue properties for the purpose of development of the Minimally Invasive Surgery (MIS) simulator [10]. We are mainly interested in the behavior of the tissues when subjected to low frequency (less than $5 \mathrm{~Hz}$ ) and large indentation stimulations, which frequently occur in real surgical practice.

In section 2 we will describe and analyze a system for measuring in vivo tissue properties. In section 3, we will show some data from animal experiments, mainly from the liver and lower esophagus of a pig. We will characterize the static and dynamic behavior of intra abdominal organs using various kinds of mathematical models in section 4. Finally, we will provide some conclusions and discussion for further study.

\section{Validation of the Measurement System}

The main idea of the measurement system for this study is to use a robotic device as a mechanical indenter because of the ease of handling and programming the motion required for the various modes of indentation. The indentation stimuli were delivered using the haptic interface device, Phantom Premium-T 1.5 (SensAble Technologies) that was programmed to perform as a mechanical stimulator. The Phantom has a nominal position resolution of $30 \mu \mathrm{m}$ and a frequency bandwidth that significantly exceeds the stimulus frequencies that were being employed in this experiment. Reaction forces were measured using a six-axis force transducer, Nano 17 (ATI Industrial Automation) that was attached to the tip of the Phantom. The transducer has a force resolution of $0.781 \mathrm{mN}$ along each of the three orthogonal axes when connected to a 16-bit A/D converter. The indenter was a $2 \mathrm{~mm}$ diameter flat-tipped cylindrical probe that was fixed to the tip of the Phantom with the force transducer mounted in-between to accurately sense the reaction forces as shown in Fig. 1(left).

Our first task was to check whether the dynamics of the device does not distort the measurements. To validate this, we developed a lumped parameter model to represent the behavior of the device both in free motion and in contact with soft tissues. Because most mechanical devices behave as low pass filters from a signal- processing viewpoint, it is important to clarify the mechanical bandwidth, which limits the dynamic range of the measurements. This model also predicts the maximum controllable 
frequency of the device without distortion of data. The model requires the parameters to represent the dynamic behavior of actuators, linkage structure and contact stiffness with soft material. In Fig. 2(b), the model consists of two concentrated masses connected by linear springs, and is a frequency varying function. The linearized model at an operating point gives a fourth-order transfer function where $\mathrm{Jm}, \mathrm{J} 1, \mathrm{kl}, \mathrm{kc}, \mathrm{N}$ are the inertia of the motor, the inertia and stiffness of the link, the stiffness of the soft tissue, and the transmission ratio, respectively. The numerical values of all parameters are listed in [11].

$$
G_{1}=\frac{F_{h}}{F_{e}}=\frac{k_{l} k_{c}}{J_{m} J_{l} s^{4}+\left(J_{m} k_{c}+J_{l} k_{l}+N^{2} J_{m} k_{l}\right) s^{2}+k_{l} k_{c}}
$$

To validate our model, we measure the frequency response of the robot using an external force transducer as in Fig 2(a). Fig. 2(c) shows the frequency response from the simulation and experiments. In the low frequency region (less than $30 \mathrm{~Hz}$ ), we can expect that the dynamics of the robot does not influence the measured data. It also shows that the structural resonance of the device is much higher than the planned indentation frequency. Therefore we conclude that the performance of the device is adequate over the frequency range of interest $(0-5 \mathrm{~Hz})$.

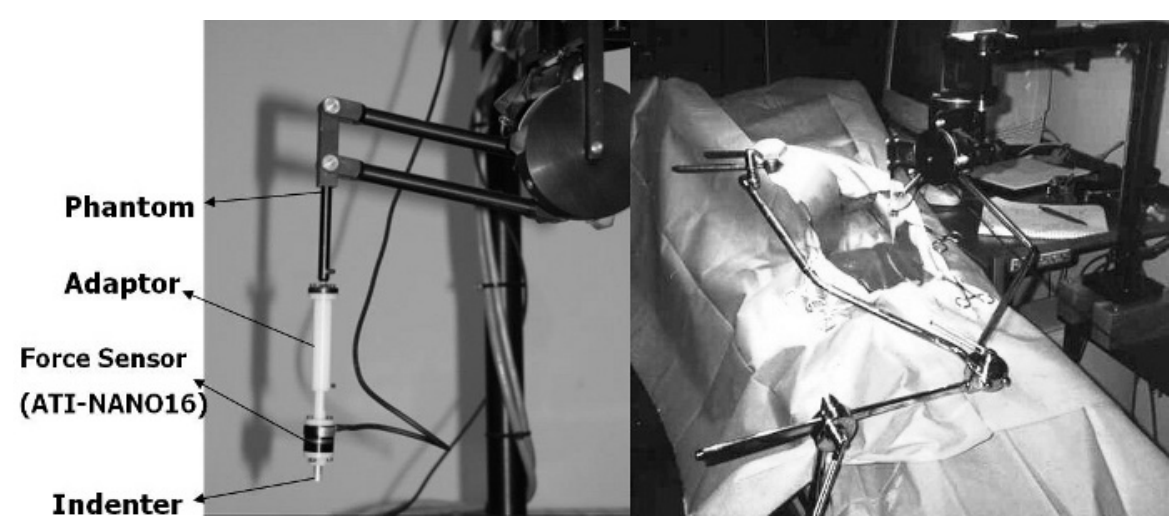

Fig. 1. (Left) Indenter consists of a phantom and a force sensor (Right) Typical view of animal experiments indenting a pig liver.

\section{In vivo Tests on Pig Intra-abdominal Tissues}

We have conducted the experiments on the liver and lower esophagus of pigs at the Harvard Center for Minimally Invasive Surgery. A typical view is shown in Fig.1(Right). The experimental protocols were approved by the MIT Committee on Animal Care as well as the Harvard Committee on Animals.

A total of 10 pigs were used in these experiments. The pigs were first put under general anesthesia and placed on the surgical table. A midline incision was then made at its abdominal region and dissection was carried out on the anatomical structures to expose the organs. The sampling rate is $1000 \mathrm{~Hz}$ both in force and displacement 
measurements. Effective management of breathing was a crucial issue when measuring the in vivo force responses. The amount of time required for each indentation stimuli and time for which the breathing of the animal was held had to be optimized. An increased period of breath holding increased the $\mathrm{CO}_{2}$ concentration in the bloodstream of the animal and caused the animal to undergo spontaneous breathing. A typical force response for ramp-and-hold input is shown Fig. 2(d). It clearly shows a viscoelastic behavior rather than a simple linear behavior. From the steady state force responses of the organs and the induced displacements, we can plot force - displacement relationships. Fig. 3 is a typical plot from the experiments.

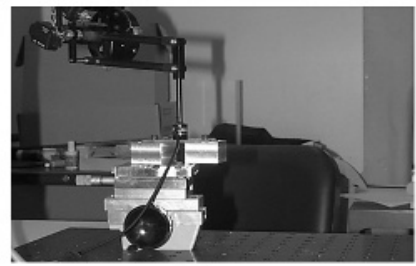

(a)

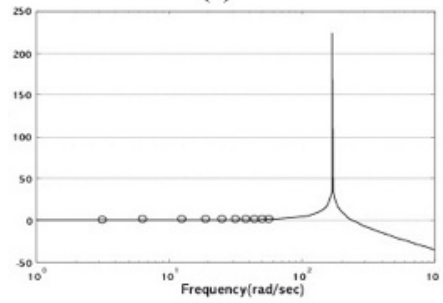

(c)

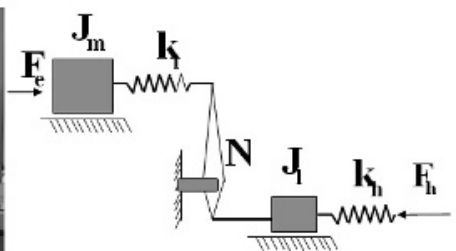

(b)

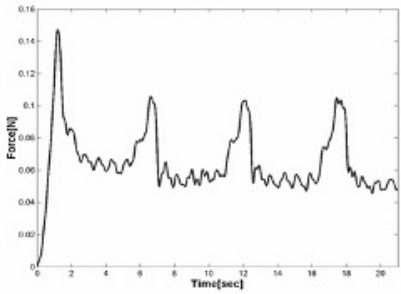

(d)

Fig. 2. (a) Experimental set up for frequency response of the measurement system. (b) The lumped model of the device (c) Frequency response of the device. Dots represent measured values and the line represents model prediction (d) Typical force response of pig liver. Large peaks are due to respiration of the animal and small peaks due to heart beats.

\section{Characterization of Tissues}

From the tissue responses to external loadings, two dominant characteristics are observed. First, the tissue responses show the nonlinearity over a certain range of displacements. Second, we can observe time-varying characteristics in the tissue responses such as stress relaxation and creep. These phenomena cannot be modeled by a simple linear elastic model. Under the assumption of isotropic, homogeneous and incompressible material, we developed a few models capturing the behavior of soft tissues for surgical simulation. One of the goals of the characterization of the tissues is to find the parameters using correlation of the mathematical model with the experimental data. These models then can be used in a finite element analysis to simulate the behavior of whole organs. 


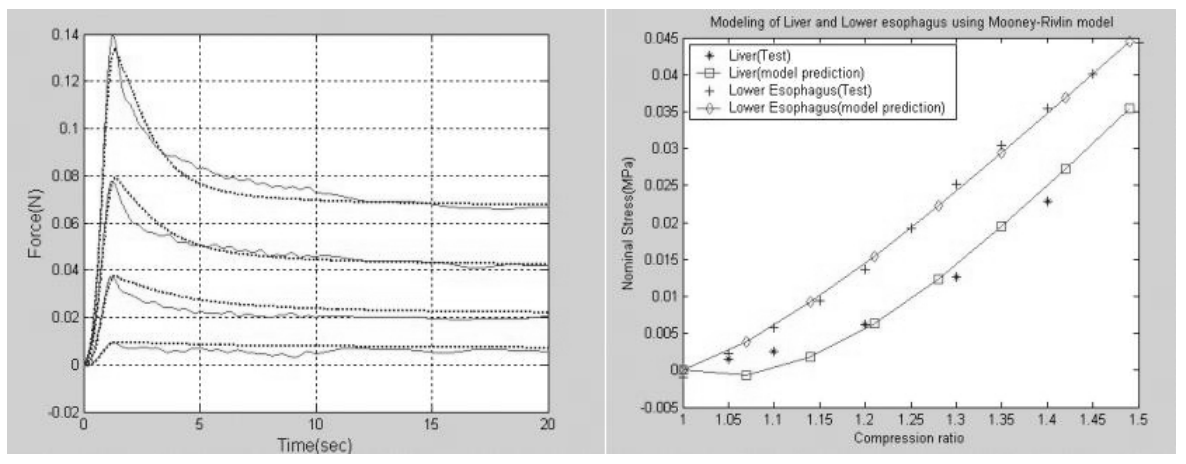

Fig. 3. (Left) Static force-displacement relationships of the liver and esophagus of pigs. (Right) The material modeling of a pig liver and lower esophagus using the blatz model.

\subsection{Estimation of the Mean Elastic Modulus}

In spite of its limitations, a linear elastic medium provides the basis of tissue modeling and many tissue models in the current simulators are based on this linear elastic theory due to its simple implementation [12]. In such a case, only the mean elastic modulus is necessary to describe the mechanical behavior of soft tissues. From the relationship of force and displacement in the case of normal indentation by a right circular indenter, the approximated mean elastic modulus can be computed by the equation (2)

$$
E=\frac{(1-v) F_{z}}{2 a \delta_{z}}
$$

E, $v, F_{z}$, a, $\delta_{z}$ are Young's modulus, Poisson's ratio, the measured force, the indenter radius, and the indented depth, respectively. Because the soft tissue is nearly incompressible in many cases, we can assume Poisson's ratio to be as close as 0.5 . The results indicate that the pig liver is more compliant than the pig lower esophagus. (For example, the Young's moduli of pig liver and lower esophagus are $31.8 \mathrm{KPa}$ and 48.8 KPa, respectively.)

\subsection{Nonlinear Static Elastic Model}

Recently, several attempts have been made to simulate nonlinear deformations of soft tissues in surgical simulations [13]. Furthermore, the addition of haptic feedback to the simulator necessitates a nonlinear tissue model for more realistic force feedback. Among the various nonlinear elastic models (nonlinear constitutive equation) developed so far, we selected an exponential material model, because the exponential curve looks very natural for describing the experimental data. One of the material models using exponential relationship is the so-called Blatz model [3] and it uses two separate exponential terms as follows, 


$$
P=\frac{\gamma}{\alpha+1}\left(\lambda e^{\alpha\left(\lambda^{2}-1\right)}-\frac{1}{\lambda^{2}} e^{\alpha(1 / \lambda-1)}\right)
$$

where $\mathrm{P}$ is the pressure at the indenter tip (force/area) and $\lambda$ is compression ratio. $\alpha, \gamma$ are two independent parameters in the model and are obtained by using least square curve fitting procedures. Fig. 3 shows that the prediction of this model matches the nonlinear response data we have obtained for the liver and lower esophagus of pigs.

\subsection{Viscoelastic Models}

When a body is deformed rapidly and then the indentation depth is maintained constant afterward, the corresponding forces decrease over time and settle to a certain value with time. This phenomenon is called stress relaxation and is one of the typical features of viscoelastic materials. Linear or nonlinear lumped parameter models are often used to predict the viscoelastic behavior of materials.

\subsubsection{Kelvin Model}

Lumped parameter models, combinations of linear springs and dampers, are widely used to describe the viscoelastic behavior of soft tissues [3]. Among them, the Kelvin model (springs in parallel with a damper) or the standard linear solid can model general viscoelastic behavior, including the stress relaxation observed in the experiments. The expression for the relaxation function $\mathrm{G}(\mathrm{t})$ can be written as

$$
G(t)=k_{1}\left[1-\left(1-\frac{k_{2}}{k_{1}}\right) e^{\frac{b}{k_{1}} t}\right] 1(t)
$$

where 1(t) is a unit step function. To determine the parameters of this assumed viscoelastic model, the experimental results on the pig liver were used. To fit the mathematical model to the experimental data, a least square optimization technique was employed. We can also use the real indentation profile without the assumption of a step input but the differences between the two cases are negligible. The estimated parameters have the following values.

$$
k_{1}=0.008(\mathrm{~N} / \mathrm{mm}), k_{2}=0.007(\mathrm{~N} / \mathrm{mm}), \mathrm{b}=0.130(\mathrm{sec} \mathrm{N} / \mathrm{mm})
$$

\subsubsection{Nonlinear Model}

Although the Kelvin model can predict the stress relaxation phenomenon, it is not capable of fully matching the force responses of a viscoelastic body. For example, the peak value of the force from the model does not match the experimental data as well as in the experiments. To Model the behavior more accurately, we use a system of nonlinear springs in parallel. 


$$
\begin{aligned}
& F=F_{0}+F_{1}+F_{2}+\ldots \\
& F_{0}=k_{01} x^{k_{02}} \\
& \left\{\begin{array}{l}
F_{1}=k_{13}\left(x-x_{1}\right)^{k_{12}} \\
\dot{x}_{1}=x_{2} \\
\dot{x}_{2}=k_{11}\left(x-x_{1}\right)^{k_{12}}-x_{2}
\end{array}\right.
\end{aligned}
$$

The parameters are determined using a nonlinear parameter estimation technique. As shown Fig. 4, the model more closely describes the viscoelastic behavior of the pig liver under various loading conditions than the single Kelvin model.

\begin{tabular}{|c|l|l|l|l|l|l|l|l|}
\hline & $\mathrm{K}_{01}$ & $\mathrm{~K}_{02}$ & $\mathrm{k}_{11}$ & $\mathrm{k}_{12}$ & $\mathrm{k}_{13}$ & $\mathrm{k}_{21}$ & $\mathrm{k}_{22}$ & $\mathrm{k}_{23}$ \\
\hline Liver & $\begin{array}{l}0.002 \\
0\end{array}$ & $\begin{array}{l}1.679 \\
7\end{array}$ & $\begin{array}{l}0.025 \\
0\end{array}$ & 2.2355 & $\begin{array}{l}0.007 \\
0\end{array}$ & 0.080 & 2.1838 & 0.0005 \\
\hline $\begin{array}{c}\text { Eso- } \\
\text { phagus }\end{array}$ & $\begin{array}{l}0.003 \\
8\end{array}$ & $\begin{array}{l}1.609 \\
7\end{array}$ & $\begin{array}{l}0.025 \\
0\end{array}$ & 1.8972 & $\begin{array}{l}0.001 \\
0\end{array}$ & 0.000 & 0.0000 & 0.0000 \\
\hline
\end{tabular}

\section{Concluding Remarks}

We have designed and built a measurement system for measuring in vivo mechanical properties of soft tissues and performed indentation experiments on the liver and lower esophagus of pigs. For characterization of the non-linear behavior of tissues, the exponential model with two independent parameters has been used. By using a nonlinear optimization technique, we can find the parameters which provide best fit model predictions with the experimental data. For characterization of the time dependent behavior, the Kelvin model and a nonlinear spring model are used.
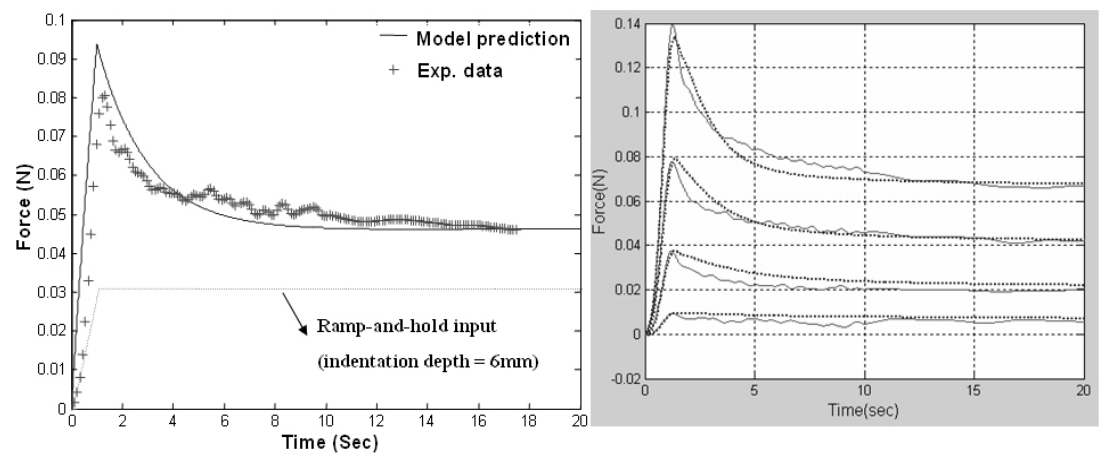

Fig. 4. Stress relaxation predicted by the Kelvin model. (Right) Various force responses predicted by the nonlinear springs matched the experimental data. 
The nonlinear spring model shows a better prediction than the Kelvin model. The material parameters obtained from the experiments can be used in combination with the geometrical dataset from the Visible Human Dataset, CT or MRI scan images of patients in medical simulations.

Acknowledgements. This work was supported by the Harvard Center for Minimally Invasive Surgery.

\section{References}

[1] R. M. Satava and S. B. Jones, "Virtual Environments for Medical Training and Education", Presence, vol. 6, pp. 139-146, 1997

[2] C. Basdogan, C. Ho, and M. A. Srinivasan, "Virtual Environments for Medical Training: Graphical and Haptic Simulation of Laparoscopic Common Bile Duct Exploration", IEEE/ASME Transactions on Mechatronics, vol. 6, pp. 269-285, 2001

[3] Y. C. Fung, Biomechanics: Mechanical Properties of Living Tissues. New York: Springer-Verlag, 1993

[4] H. Yamada, Strength of Biological Materials. Baltimore: Williams and Wilkins Co., 1970.

[5] J. Rosen, B. Hannaford, M. MacFarlane, and M. Sinanan, "Force Controlled and Teleoperated Endoscopic Grasper for Minimally Invasive Surgery - Experimental Performance Evaluation", IEEE Transactions on Biomedical Engineering, vol. 46, pp. 1212-1221, 1999

[6] F. J. Carter, T. G. Frank, P. J. Davies, D. Mclean, and A. Cuschieri, "Biomechanical Testing on Intra-abdominal Soft Tissues", Medical Image Analysis, 2000.

[7] I. Brouwer, J. Ustin, L. Bentley, A. Sherman, N. Dhruv, and F. Tendick, "Measuring In Vivo Animal Soft Tissue Properties for Haptic Modeling in Surgical Simulation", Proceedings of MMVR Conference, 2001

[8] R. J. Gulati and M. A. Srinivasan, "Determination of Mechanical Properties of the Human Fingerpad, In Vivo, Using a Tactile Stimulator," MIT, Touch Lab RLE TR-605, 1996

[9] M. P. Ottensmeyer, "In vivo Measurement of Solid Organ Viscoelastic Properties", Proceedings of MMVR Conference, 2002

[10] B. K. Tay, S. De, N. Stylopoulos, D. W. Rattner, and M. A. Srinivasan, "In vivo Force Response of Intra-abdominal Soft Tissue for the Simulation of Laparoscopic Procedures" Proceedings of the MMVR Conference, 2002

[11] M. C. Cavusoglu, D. Feygin, and F. Tendick, "A Critical Study of the Mechanical and Electrical Properties of the PHANToM Haptic Interface and Improvements for High Performance Control", Presence: Teleoperators and Virtual Environments, vol. 11, 2002

[12] S. Cotin, H. Delingette, and N. Ayache, "Real-time elastic deformations of soft tissue for surgery simulation", IEEE Trans. On Visualization and computer graphics, vol. 5, pp. 62-73, 1999

[13] X. Wu, M. S. Downes, T. Goktekin, and F. Tendick, "Adaptive Nonlinear Finite Elements for Deformable Body Simulation Using Dynamic Progressive Meshes," Computer Graphics Forum, vol. 20, pp. 349-58, 2001 